\title{
Erythropoietin Pretreatment of Transplanted Endothelial Colony-Forming Cells Enhances Recovery in a Cerebral Ischemia Model by Increasing Their Homing Ability: A SPECT/CT Study
}

\author{
Philippe Garrigue ${ }^{1-3}$, Guillaume Hache ${ }^{1}$, Youssef Bennis ${ }^{1}$, Pauline Brige ${ }^{4}$, Jimmy Stalin ${ }^{1}$, Lionel Pellegrini ${ }^{1}$, \\ Lionel Velly ${ }^{5}$, Francesca Orlandi ${ }^{6}$, Elena Castaldi ${ }^{6}$, Françoise Dignat-George ${ }^{1,7}$, Florence Sabatier ${ }^{1,8}$, \\ and Benjamin Guillet ${ }^{1-3}$ \\ ${ }^{1}$ UMR_S 1076, INSERM, Aix Marseille Univ., Marseille, France; ${ }^{2}$ CERIMED, Aix Marseille Univ., Marseille, France; ${ }^{3}$ Service \\ Radiopharmacie, Hôpital Nord, APHM, Marseille, France; ${ }^{4}$ LIIE, Aix Marseille Univ., Marseille, France; ${ }^{5}$ Département Anesthésie- \\ Réanimation Adulte, APHM, Marseille, France; ${ }^{6}$ Advanced Accelerator Applications, Colleretto-Giacosa, Italy; ${ }^{7}$ Service \\ d'Hématologie, Hôpital Conception, APHM, Marseille, France; and ${ }^{8}$ Laboratoire de Culture et Thérapie Cellulaire, Hôpital \\ Conception, APHM, Marseille, France
}

Endothelial colony-forming cells (ECFCs) are promising candidates for cell therapy of ischemic diseases, as less than $10 \%$ of patients with an ischemic stroke are eligible for thrombolysis. We previously reported that erythropoietin priming of ECFCs increased their in vitro and in vivo angiogenic properties in mice with hindlimb ischemia. The present study used SPECT/CT to evaluate whether priming of ECFCs with erythropoietin could enhance their homing to the ischemic site after transient middle cerebral artery occlusion (MCAO) followed by reperfusion in rats and potentiate their protective or regenerative effect on blood-brain barrier (BBB) disruption, cerebral apoptosis, and cerebral blood flow (CBF). Methods: Rats underwent a 1-h MCAO followed by reperfusion and then $1 \mathrm{~d}$ after MCAO received an intravenous injection of either PBS (control, $n$ $=10$ ), PBS-primed ECFCs (ECFC PBS, $_{n=13}$ ), or erythropoietinprimed ECFCs (ECFC EPO $_{n} n=10$ ). ECFC homing and the effect on BBB disruption, cerebral apoptosis, and CBF were evaluated by SPECT/CT up to $14 \mathrm{~d}$ after MCAO. The results were expressed as median \pm interquartile range for ipsilateral-to-contralateral ratio of the activity in middle cerebral artery-vascularized territories in each hemisphere. Histologic evaluation of neuronal survival and astrocytic proliferation was performed on day 14. Results: Erythropoietin priming increased homing of ECFCs to the ischemic hemisphere (ECFC ${ }_{\mathrm{PBS}}$, $111.0 \% \pm 16.0 \%$; ECFC $_{\text {EPO }}, 146.5 \% \pm 13.3 \%$ ). BBB disruption was significantly reduced (control, $387 \% \pm 153 \%$; ECFC EBS $_{\mathrm{PBS}}, 151 \% \pm$ $46 \%[P<0.05]$; ECFC negatively with ECFC homing (Pearson $r=-0.6930, P=0.0002$ ). Cerebral apoptosis was significantly reduced (control, $161 \% \pm 10 \%$; ECFC $\left._{\mathrm{PBS}}, 141 \% \pm 9 \%[P<0.05] ; \mathrm{ECFC}_{\mathrm{EPO}}, 118 \% \pm 5 \%[P<0.001]\right)$ and correlated negatively with ECFC homing $(r=-0.7251, P<$ 0.0001). CBF was significantly restored with ECFCs and almost totally so with erythropoietin priming (control, $72 \% \pm 2 \%$; ECFC $_{\mathrm{PBS}}, 90 \% \pm$ $4 \%[P<0.01]$; ECFC EPO $\left._{1}, 99 \% \pm 4 \%[P<0.001]\right)$ and correlated positively with ECFC homing $(r=0.7348, P<0.0001)$. Immunoblocking against the CD146 receptor on ECFCs highlighted its notable role

Received Dec. 3, 2015; revision accepted May 20, 2016.

For correspondence or reprints contact: Benjamin Guillet, Faculté de Pharmacie, 27 Boulevard Jean Moulin, 13005 Marseille, France.

E-mail: benjamin.guillet@univ-amu.fr

Published online Sep. 8, 2016.

COPYRIGHT (C) 2016 by the Society of Nuclear Medicine and Molecular Imaging, Inc. in ECFC homing with erythropoietin priming $\left(\mathrm{ECFC}_{\mathrm{EPO}}, 147 \% \pm\right.$

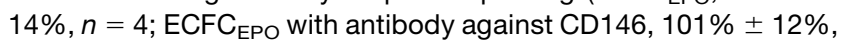
$n=4[P<0.05])$. Conclusion: Priming with erythropoietin before cell transplantation is an efficient strategy to amplify the migratory and engraftment capacities of ECFCs and their beneficial impact on BBB disruption, apoptosis, and CBF.

Key Words: ischemic stroke; blood-brain barrier; apoptosis; cerebral blood flow; ECFC; homing

J Nucl Med 2016; 57:1798-1804

DOI: 10.2967/jnumed.115.170308

T schemic stroke is a leading cause of morbidity and mortality with a constantly increasing prevalence worldwide (1). New approaches including neuroprotective or neurorestorative therapies are still under evaluation. Among them, stem cell therapy-more specifically, endothelial progenitor cell transplantation-is considered one of the most promising strategies. Endothelial progenitor cells are endogenous immature cells mobilized from bone marrow that have the ability to home to the site of recovering tissue after ischemic injury (2). Endothelial colony-forming cells (ECFCs) are of particular interest because they constitute a homogeneous and well-characterized endothelial progenitor cell population displaying an ability to form vessels de novo (3). We previously reported that ECFC transplantation in rats after middle cerebral artery occlusion (MCAO) attenuated neurologic deficits by decreasing acute neurodegeneration and enhancing tissue regeneration, although homing and engraftment of ECFCs into the ischemic tissue appeared relatively poor after intravenous delivery (4). Thus, new approaches to enhancing the effect of stem cell transplantation are desirable for regenerative treatment of stroke: we recently stated that the well-known pretreatment (5) or cotreatment (6) of ECFCs with pharmaceutical-grade erythropoietin increased their in vitro and in vivo angiogenic properties. In parallel, new, effective tools are needed to evaluate their benefits, and we recently reported the SPECT characterization of cerebral blood flow $(\mathrm{CBF})$, blood-brain barrier (BBB) disruption, and cerebral apoptosis up to $14 \mathrm{~d}$ after MCAO in rats (7). 
The present study aimed to evaluate whether erythropoietin priming of ECFCs could enhance their homing to the ischemic site after $\mathrm{MCAO}$ in rats and potentiate their protective or regenerative effect on BBB disruption, cerebral apoptosis, and CBF.

\section{MATERIALS AND METHODS}

The study was approved by the Animal Care Committee of AixMarseille Université (ethical committee CE14) and was conducted according to European Union Directive 2010/63/EU and the recommendations of the Helsinki Declaration. Experiments were performed in an authorized laboratory (13C-055-20) by trained and competent researchers. A masked experimenter performed the neurologic evaluations and histologic quantifications.

\section{MCAO Induction}

Adult male Sprague-Dawley rats (Janvier-Labs) weighing 230$280 \mathrm{~g}$ were used in all experiments. The rats were intubated and the lungs mechanically ventilated with 3.0 volume percent sevoflurane in a gas mixture of $30 \%$ oxygen and $70 \%$ nitrogen. During anesthesia, the inspired and expired fractions of sevoflurane, oxygen, and carbon dioxide were continuously monitored (Capnomac Ultima; Datex-Ohmeda). A 60-min MCAO was induced as previously described (7).

\section{Cell Preparation and Priming}

Human umbilical cord blood samples from healthy donors were collected in compliance with French legislation, and ECFCs were isolated as previously reported (8).

Priming consisted of incubating the ECFCs for $24 \mathrm{~h}$ with human recombinant erythropoietin (epoetin- $\alpha$ [Eprex; Janssen-Cilag], 10,000 $\mathrm{IU} / \mathrm{mL}$ ) to a final concentration of $5 \mathrm{IU} / \mathrm{mL}$ diluted in endothelial basal medium 2 (Lonza) supplemented with $0.5 \%$ fetal bovine serum. After extensive washing, the cells $\left(\mathrm{ECFC}_{\mathrm{EPO}}\right)$ were used in subsequent assays. Cells maintained in $0.5 \%$ fetal bovine serum/endothelial basal medium 2 and pretreated with phosphate-buffered saline (PBS; Lonza) instead of erythropoietin for $24 \mathrm{~h}$ were used as control cells $\left(\mathrm{ECFC}_{\mathrm{PBS}}\right)$. In blocking experiments, antibody against CD146 (anti-CD146 S-endo 1 clone [Biocytex], $1 \mu \mathrm{g} / \mathrm{mL}$ of medium) was added after erythropoietin or PBS incubation for $1 \mathrm{~h}\left(\mathrm{ECFC}_{\mathrm{EPO}+\mathrm{aCD146}}\right.$ or $\mathrm{ECFC}_{\mathrm{PBS}+\mathrm{aCD} 146}$, respectively).

Twenty-four hours after MCAO, animals were randomly allocated to PBS injection, $\mathrm{ECFC}_{\mathrm{PBS}}$, or $\mathrm{ECFC}_{\mathrm{EPO}}$ using an automated research randomizer available at http://www.randomizer.org. For blocking experiments, animals were randomly allocated to $\mathrm{ECFC}_{\mathrm{PBS}}, \mathrm{ECFC}_{\mathrm{PBS}+\mathrm{aCD} 146}$, $\mathrm{ECFC}_{\mathrm{EPO}}$, or $\mathrm{ECFC}_{\mathrm{EPO}+\mathrm{aCD} 146}$. PBS alone $(0.2 \mathrm{~mL})$ or $4 \times 10^{6} \mathrm{ECFCs}$ in PBS $(0.2 \mathrm{~mL})$ were delivered via the tail vein of the control and transplantation animals, respectively (Fig. 1).

To assess a putative toxic effect of CD146 immunoblocking, we evaluated cell survival using the 7-AAD annexin- $\mathrm{V}$ fluorescein isothiocyanate kit (Beckman-Coulter). ECFCs were plated in 6-well plates and exposed to $0.5 \%$ fetal bovine serum/endothelial basal medium 2 with anti-CD146 antibody $(1 \mu \mathrm{g} / \mathrm{mL})$ for $1 \mathrm{~h}$. Samples were interpreted in a FC500 flow cytometer (Beckman-Coulter). Cells that showed low staining for both annexin- $\mathrm{V}$ and $7-\mathrm{AAD}$ were considered prosurvival cells.

\section{Radiotracers and Radiolabeling}

Hexamethylpropyleneamine-oxime (HMPAO [Cerestab]) and pentetic acid (DTPA [Pentacis]) were purchased from, respectively, GE Healthcare and IBA. The experimental annexin V-128 kit was provided by Advanced Accelerator Applications. Radiolabeling with fresh ${ }^{99} \mathrm{~m} \mathrm{TcO}_{4}{ }^{-}$pertechnetate (physical half-life, $6 \mathrm{~h}$; $\gamma$-energy, 140 $\mathrm{keV}$ ) was performed according to the manufacturer's instructions. Radiochemical purities were confirmed to be at least $95 \%$ by thinlayer chromatography.

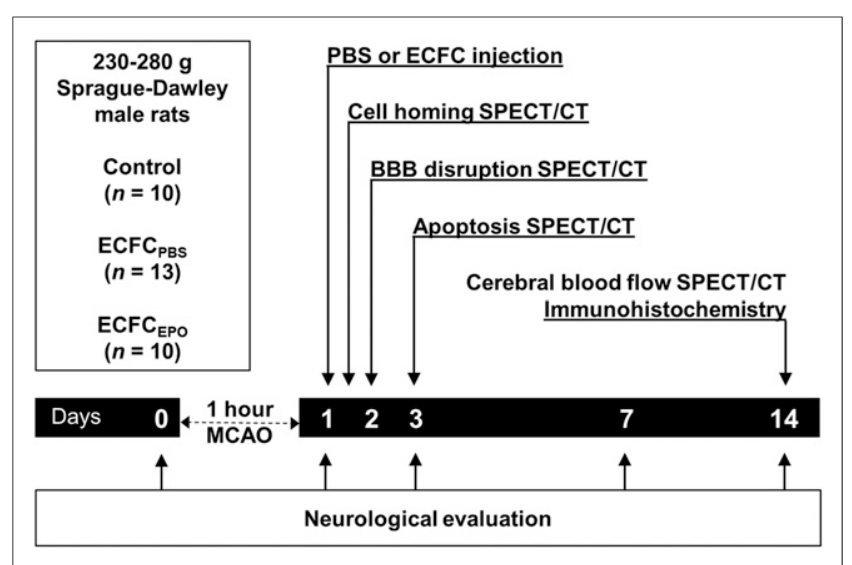

FIGURE 1. Experimental protocol. Rats underwent MCAO and then were treated with PBS (control), ECFC PBS, or ECFC $_{\text {EPO }} 1 \mathrm{~d}$ afterward. Twelve hours after injection of the radiolabeled cells, ECFC homing was assessed using SPECT/CT. Two, 3, and $14 \mathrm{~d}$ after MCAO, BBB disruption was assessed using 99mTc-DTPA, apoptosis using 99mTc-annexin$\mathrm{V}-128$, and CBF using 99mTc-HMPAO, respectively. Fourteen days after $\mathrm{MCAO}$, neuronal survival and astrocyte proliferation were evaluated ex vivo using immunohistochemistry.

\section{ECFC Radiolabeling and Labeling with CM-Dil}

Radioactive labeling of cells was performed as reported by Detante et al. (9). Briefly, $40 \times 10^{6}$ ECFCs were trypsinized into a single-cell suspension and then mixed with $370 \mathrm{MBq}$ of ${ }^{99 \mathrm{~m} T c-H M P A O}$ for $30 \mathrm{~min}$ at $37^{\circ} \mathrm{C}$ and $5 \% \mathrm{CO}_{2}$. The cells were centrifuged and washed twice with PBS and counted. Labeling efficiency was $68 \% \pm 7 \%$ of total radioactivity. For the immunoblocking experiment, a colabeling was realized by incubating cells with $5 \mu \mathrm{L}$ of CM-DiI (Vybrant; Life Technologies) per milliliter of suspension for $20 \mathrm{~min}$ at $37^{\circ} \mathrm{C}$ and $5 \%$ $\mathrm{CO}_{2}$. ECFCs were washed twice with PBS and resuspended in $3 \mathrm{~mL}$ of PBS at $37^{\circ} \mathrm{C}$, and $4 \times 10^{6}$ labeled ECFCs $(25 \pm 3 \mathrm{MBq})$ were injected into the tail vein $24 \mathrm{~h}$ after MCAO.

\section{Assessment of CD146 Expression in \\ Erythropoietin-Primed ECFCs}

Thirty micrograms of $\mathrm{ECFC}_{\mathrm{PBS}}$ and $\mathrm{ECFC}_{\mathrm{EPO}}$ cell lysates (lysis buffer: Tris, $10 \mathrm{mM}, \mathrm{pH} 8$; ethylenediaminetetraacetic acid, $1 \mathrm{mM}$, $\mathrm{pH} 8 ; \mathrm{NaCl}, 150 \mathrm{mM}$; NP40, 1\%; and proteases inhibitors) were subjected to $4 \%-12 \%$ NuPAGE SDS-polyacrylamide gel electrophoresis (Life Technologies) and transferred onto a nitrocellulose membrane with an IBLOT transfer device (Life Technologies). Membranes were incubated with BSA (4\%), anti-CD146 antibody (clone 7A4; Biocytex), antiactin (CellSignaling), and a secondary peroxidase-coupled antibody before detection with enhanced chemiluminescence (Amersham). CD146 membrane expression was also determined on ECFCs by flow cytometry. Cells were labeled with phycoerythrin-conjugated antibodies (anti-CD146 S-endo 1 antibody or isotype-matched IgG as negative control) for $45 \mathrm{~min}$ at $4^{\circ} \mathrm{C}$. After washing, samples were analyzed by flow cytometry on a Beckman Coulter apparatus (FC500). The number of antigenic sites per cell was counted using a quantitative indirect immunofluorescence assay based on the linear relation between antigen expression and the median fluorescence intensity. Results are expressed as percentage of control.

\section{SPECT Data Acquisition}

According to the experimental paradigm (Fig. 1), ${ }^{99} \mathrm{~m}$ Tc-DTPA $(20 \mathrm{MBq} / 100 \mu \mathrm{L}),{ }^{99 \mathrm{~m} T c}$-annexin-V-128 (37 MBq/150 $\left.\mu \mathrm{L}\right)$, and ${ }^{99 \mathrm{~m} T c-H M P A O}(20 \mathrm{MBq} / 150 \mu \mathrm{L})$ was injected through the tail vein on days 2, 3, and 14, respectively, after MCAO. The animals were 
imaged under a NanoSPECT/CT ${ }^{\text {PLUS }}$ camera (Bioscan) $30 \mathrm{~min}$ after ${ }^{99 \mathrm{~m}} \mathrm{Tc}-\mathrm{DTPA}$ or ${ }^{99 \mathrm{~m}} \mathrm{Tc}-\mathrm{HMPAO}$ injection and $90 \mathrm{~min}$ after ${ }^{99 \mathrm{~m}} \mathrm{Tc}$-annexin-V-128 injection. Multipinhole SPECT parameters were set up as follows: termination condition, 10,000 counts; picture size, $256 \times 256$; zoom factor, 1.14 ; and pixel size, $1.00 \mathrm{~mm}^{2}$.

\section{SPECT Image Analysis}

Images were reconstructed and analysis was performed using the 3dimensional region-of-interest module of InVivoScope software, version $2.0 \mathrm{p} 4$ (inviCRO). After coregistration between SPECT and $\mathrm{CT}, 2$ volumes of interest were drawn according to the middle cerebral artery-vascularized territories in each hemisphere, with the CT overlay images providing anatomic guidance. Radioactivity inside each volume of interest was quantified and corrected by tissue volume $\left(\mathrm{MBq} / \mathrm{mm}^{3}\right)$. We then calculated the ipsilateral-to-contralateral (I/C) ratios. Image color scales were normalized for comparing signals between conditions.

\section{Measurement of Neurologic Outcome}

Neurologic deficits were evaluated on days 1, 3, 7, and 14 after MCAO. Neurologic function was graded using the modified neurologic severity score (10) (normal score, 0; maximal deficit score, 18). Somatosensory deficit was measured both before and after MCAO with the adhesive-removal somatosensory test. The time necessary to remove a small adhesive-backed paper dot from the limb was recorded during 3 trials per day for $3 \mathrm{~d}$ before MCAO and on days $1,3,7$, and 14. Sensorimotor function was evaluated by the foot-fault test. The rats were allowed to walk on a horizontal 77-bar scale. A computer-assisted device (Locotronic; Bioseb) was used to record foot faults (falling or slipping of paws between bars). Each animal performed the test 3 times per day for $3 \mathrm{~d}$ before MCAO then on days 1 , 3,7 , and 14 after MCAO.

\section{Immunohistochemistry}

The animals were euthanized with a lethal dose of pentobarbital (Clin-Midy) $2 \mathrm{~d}$ (blocking experiment) or $14 \mathrm{~d}$ after MCAO and perfused by a $4 \%$ phosphate-buffered paraformaldehyde solution (Sigma-Aldrich). The brains were harvested, postfixed for $24 \mathrm{~h}$, cryopreserved in $10 \%$ and $30 \%$ sucrose, snap-frozen, and stocked at $-80^{\circ} \mathrm{C}$. Frozen sections were cut with a sliding microtome (CM1900; Leica). Immunohistochemical labeling was performed using primary anti-GFAP (1:100, Dako) and anti-NeuN (1:1,000, Millipore) monoclonal antibodies and then was detected with Bond Polymer Refine Red Detection and Bond Polymer Refine HRP Detection kits (Leica). Five fields of view were quantified in the ipsi- and contralateral hemispheres for each slide under a light and fluorescent microscope
(Eclipse-Ni; Nikon) at $\times 2$ and $\times 10$ magnifications using thresholdbase labeling detection after saturation and intensity calibration. The results are expressed as the NeuN- or GFAP-positive-hit I/C ratio.

\section{Statistical Analysis}

Values are expressed as median \pm interquartile range unless otherwise indicated. Statistical differences were analyzed using KruskalWallis ANOVA with Dunn post hoc analysis when comparing 3 groups and Mann-Whitney testing when comparing 2 groups. Pearson correlation was assessed between ECFC homing and BBB disruption, apoptosis, or CBF SPECT/CT quantifications. Statistical analyses were performed with Prism (GraphPad Software), with a $P$ value of less than 0.05 being considered statistically significant.

\section{RESULTS}

The modified neurologic severity scores were significantly lower in both $\mathrm{ECFC}_{\mathrm{PBS}}$ and $\mathrm{ECFC}_{\mathrm{EPO}}$ than in control on day 7 (control, $7.1 \pm 3.7, n=10 ; \mathrm{ECFC}_{\mathrm{PBS}}, 2.7 \pm 2.2, n=13[P<0.001]$; $\mathrm{ECFC}_{\mathrm{EPO}}, 2.0 \pm 2.6, n=10[P<0.001]$ ) and day 14 (control, $7.7 \pm 5.7, n=10 ; \mathrm{ECFC}_{\mathrm{PBS}}, 2.6 \pm 3.0, n=13[P<0.001]$; $\mathrm{ECFC}_{\mathrm{EPO}}, 1.4 \pm 2.1, n=10[P<0.001]$ ) (Fig. 2A).

Compared with control, sensorimotor function was significantly improved in both $\mathrm{ECFC}_{\mathrm{PBS}}$ and $\mathrm{ECFC}_{\mathrm{EPO}}$ on day 7 (control, $25.4 \pm$ 21.0, $n=10 ; \mathrm{ECFC}_{\mathrm{PBS}}, 9.9 \pm 13.8, n=13[P<0.01] ; \mathrm{ECFC}_{\mathrm{EPO}}$, $5.6 \pm 2.6, n=10[P<0.001])$ and day 14 (control, $23.7 \pm 20.1$, $n=10 ; \mathrm{ECFC}_{\mathrm{PBS}}, 7.3 \pm 3.5, n=13[P<0.001] ; \mathrm{ECFC}_{\mathrm{EPO}}, 4.6 \pm$ 4.2, $n=10[P<0.001])$ (Fig. 2B).

The time to remove the adhesive (Fig. 2C) was significantly shorter in both $\mathrm{ECFC}_{\mathrm{PBS}}$ and $\mathrm{ECFC}_{\mathrm{EPO}}$ than in control on day 7 (control, $111.6 \pm 75 \mathrm{~s}, n=10$; $\mathrm{ECFC}_{\mathrm{PBS}}, 29.3 \pm 29 \mathrm{~s}, n=13[P<$ 0.001]; $\mathrm{ECFC}_{\mathrm{EPO}}, 25.5 \pm 43 \mathrm{~s}, n=10$ ) and day 14 (control, $101.4 \pm$ $70 \mathrm{~s}, n=10$; $\mathrm{ECFC}_{\mathrm{PBS}}, 20.9 \pm 34 \mathrm{~s}, n=13[P<0.001]$; $\mathrm{ECFC}_{\mathrm{EPO}}$, $22.3 \pm 24 \mathrm{~s}, n=10[P<0.001])$. On day 3 , the adhesive removal time was significantly shorter in $\mathrm{ECFC}_{\mathrm{EPO}}$, but not in $\mathrm{ECFC}_{\mathrm{PBS}}$, than in control (control, $26.8 \pm 19 \mathrm{~s}, n=10 ; \mathrm{ECFC}_{\mathrm{PBS}}, 23.3 \pm 20 \mathrm{~s}, n=$ $13[P=$ not statistically significant $] ; \mathrm{ECFC}_{\mathrm{EPO}}, 15.6 \pm 12 \mathrm{~s}, n=10$ $[P<0.001])$.

\section{Erythropoietin-Primed ECFCs Enhanced Neuron Survival and Decreased Astrocyte Proliferation}

Fourteen days after MCAO, we found a significantly higher NeuNpositive-hit $\mathrm{I} / \mathrm{C}$ ratio in $\mathrm{ECFC}_{\mathrm{PBS}}(65 \% \pm 12 \%, n=13)$ than in control $(40 \% \pm 5 \%, n=10[P<0.05])$, and the NeuN-positive-hit
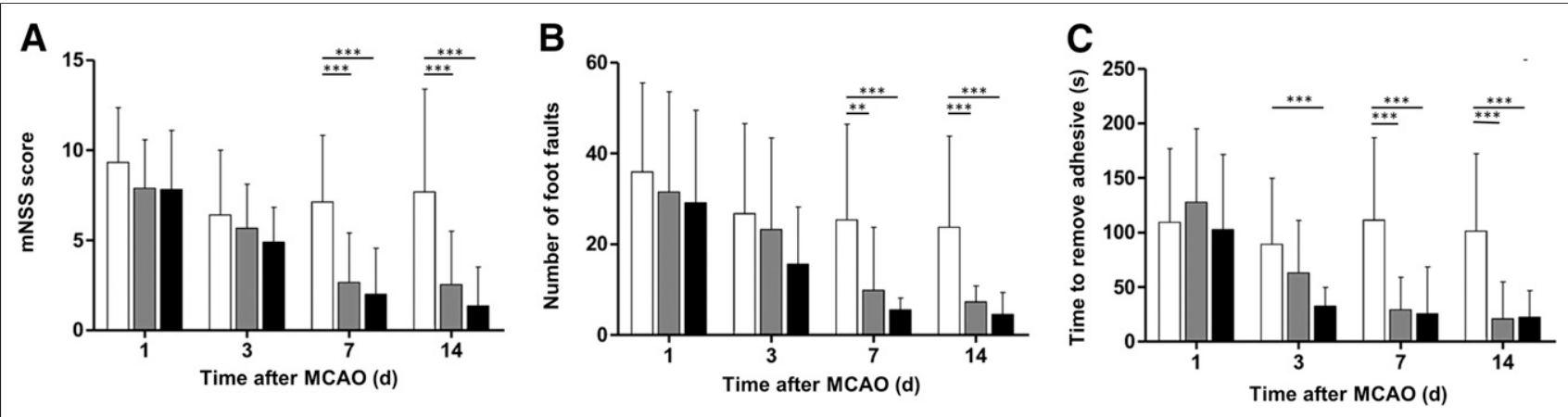

FIGURE 2. Effect of ECFC treatment on neurologic evaluations. Evolution of modified neurologic severity score (A), foot-fault number (B), and time to remove adhesive tape (C) for control (white bars), ECFC $C_{P B S}$ (gray bars), and ECFC $E_{E P O}$ (black bars) are graphed for up to $14 \mathrm{~d}$ after MCAO. ${ }^{* \star} P<$ 0.01 . ${ }^{\star \star \star} P<0.001$. 


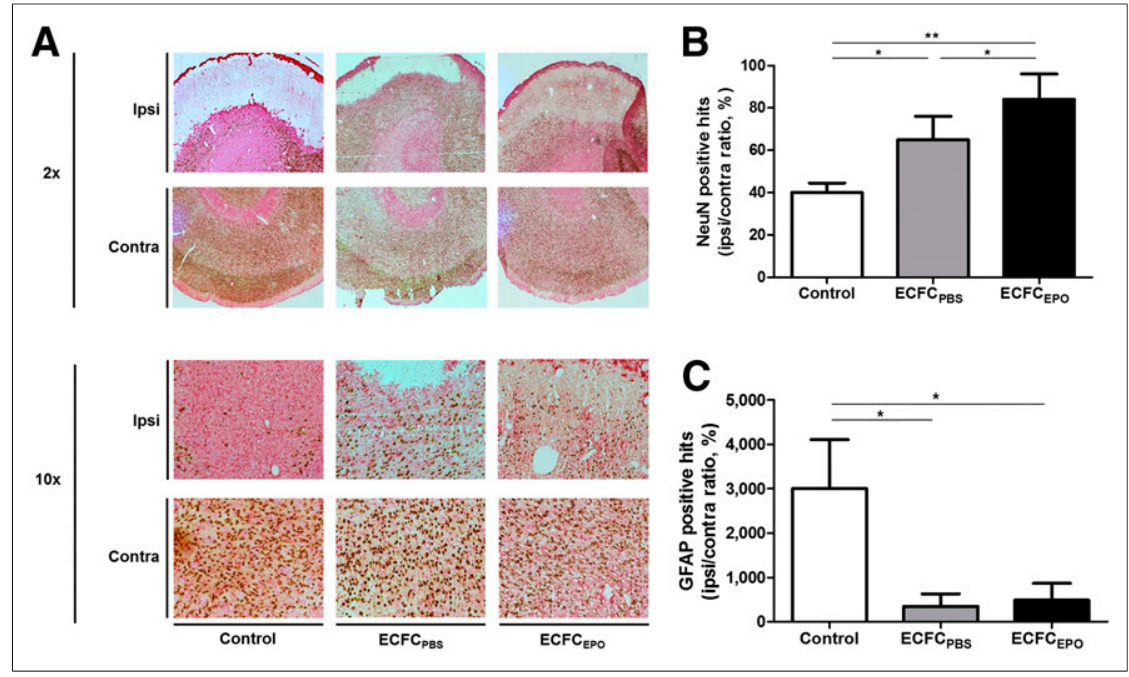

FIGURE 3. Effect of ECFC treatment on neuronal survival and astrocytic proliferation. (A) Brain sections of control, ECFC $C_{P B S}$, and $E_{C F} C_{E P O}$ as examined under light microscope at $\times 2$ and $\times 10$ magnification after NeuN/GFAP staining $14 \mathrm{~d}$ after MCAO. (B and C) Median \pm interquartile range for NeuN-positive hits (B) and GFAP-positive hits (C). ${ }^{*} P<0.05 .{ }^{* *} P<0.01$.

$\mathrm{I} / \mathrm{C}$ ratio was even higher in $\mathrm{ECFC}_{\mathrm{EPO}}(84 \% \pm 10 \%, n=10)$ than in control $(P<0.01)$ or $\mathrm{ECFC}_{\mathrm{PBS}}(P<0.05)$ (Figs. 3A and $\left.3 \mathrm{~B}\right)$. Similarly, we found a significantly higher GFAP-positive-hit I/C ratio in control $(3,000 \% \pm 1,171 \%, n=10)$ than in $\mathrm{ECFC}_{\mathrm{PBS}}(349.5 \% \pm$ $365.8 \%, n=13)$ or $\mathrm{ECFC}_{\mathrm{EPO}}(484 \% \pm 395 \%, n=10[P<0.05])$ (Figs. 3A and 3C).

\section{Erythropoietin Priming Increased ECFC Homing to Ischemic Territory}

SPECT/CT $12 \mathrm{~h}$ after radiolabeled ECFC injection showed a tight $\mathrm{I} / \mathrm{C}$ ratio for $\mathrm{ECFC}_{\mathrm{PBS}}(111.0 \% \pm 16.0 \%, n=13)$, and this ratio was significantly increased to $146.5 \% \pm 13.3 \%$ with $\mathrm{ECFC}_{\mathrm{EPO}}$ ( $n=10[P<0.0001])$ (Fig. 4A).

\section{Erythropoietin Priming Induced CD146 Overexpression in ECFCs}

Western blotting and flow cytometry experiments showed that erythropoietin priming induced a significantly increased
CD146 expression for ECFCs (respectively, $154 \% \pm 43 \%$ and $108 \% \pm 7 \%$ vs. control, both $n=3$ [both $P<0.05$ ]) (Figs. 4B and 4C).

\section{CD146 Immunoblocking Abolished Erythropoietin-Primed ECFC Homing}

Although no significant difference in $\mathrm{I} / \mathrm{C}$ ratio was found between $\mathrm{ECFC}_{\mathrm{PBS}}(119 \% \pm$ $10 \%, n=3)$ and $\mathrm{ECFC}_{\mathrm{PBS}+\mathrm{aCD} 146}(118 \% \pm$ $6 \%, n=3$ ) (Fig. 5A), CD146 immunoblocking induced a significant decrease in transplanted ECFC homing ability after erythropoietin preconditioning, from $147 \% \pm$ $14 \%(n=4)$ for $\mathrm{ECFC}_{\mathrm{EPO}}$ to $101 \% \pm$ $12 \%(n=4)$ for $\mathrm{ECFC}_{\mathrm{EPO}+\mathrm{aCD} 146}(P<$ $0.05)$ (Fig. 5B). CD146 immunoblocking did not affect cell survival $12 \mathrm{~h}$ after incubation $(95 \% \pm 4 \%)$ (Fig. 5C).

Immunofluorescence showed no significant difference in the CM-DiI-positive I/C ratio between $\mathrm{ECFC}_{\mathrm{PBS}}(119.0 \% \pm 10.5 \%$, $n=3)$ and $\mathrm{ECFC}_{\mathrm{PBS}+\mathrm{aCD} 146}(118.0 \% \pm$ $6.0 \%, n=3$ ) (Fig. 5E), whereas this ratio was significantly decreased in $\mathrm{EFCF}_{\mathrm{EPO}+\mathrm{aCD} 146}(101.5 \% \pm 11.5 \%, n=4)$ compared with $\operatorname{ECFC}_{\mathrm{EPO}}(146.5 \% \pm 13.5 \%, n=4[P<0.01])$ (Figs. 5D and 5F).

\section{ECFC Homing Potentiation Affects BBB Disruption, Cerebral Apoptosis, and CBF SPECT Imaging}

Two days after MCAO, the ${ }^{99 \mathrm{~m}} \mathrm{Tc}-\mathrm{DTPA} \mathrm{I} / \mathrm{C}$ ratio was significantly decreased in $\mathrm{ECFC}_{\mathrm{PBS}}(151 \% \pm 46 \%, n=13[P<0.05])$ and $\operatorname{ECFC}_{\mathrm{EPO}}(112 \% \pm 9 \%, n=10[P<0.001])$, compared with

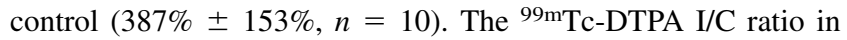
$\mathrm{ECFC}_{\mathrm{EPO}}$ was significantly lower than that in $\mathrm{ECFC}_{\mathrm{PBS}}(P<$ $0.05)$. BBB disruption was significantly and inversely correlated to ECFC homing ( $r=-0.6930, P=0.0002)$ (Fig. 6A).

Three days after MCAO, the ${ }^{99 \mathrm{~m}} \mathrm{Tc}$-annexin- $\mathrm{V}-128 \mathrm{I} / \mathrm{C}$ ratio was lower in $\mathrm{ECFC}_{\mathrm{PBS}}(141 \% \pm 9 \%, n=13[P<0.05])$ and $\mathrm{ECFC}_{\mathrm{EPO}}$ $(118 \% \pm 5 \%, n=10[P<0.001])$ than in control $(161 \% \pm 10 \%$, $n=10)$. The ${ }^{99 \mathrm{~m}} \mathrm{Tc}$-annexin- $\mathrm{V}-128 \mathrm{I} / \mathrm{C}$ ratio in $\mathrm{ECFC}_{\mathrm{EPO}}$ was significantly lower than that in $\operatorname{ECFC}_{\mathrm{PBS}}(P<0.05)$. Cerebral
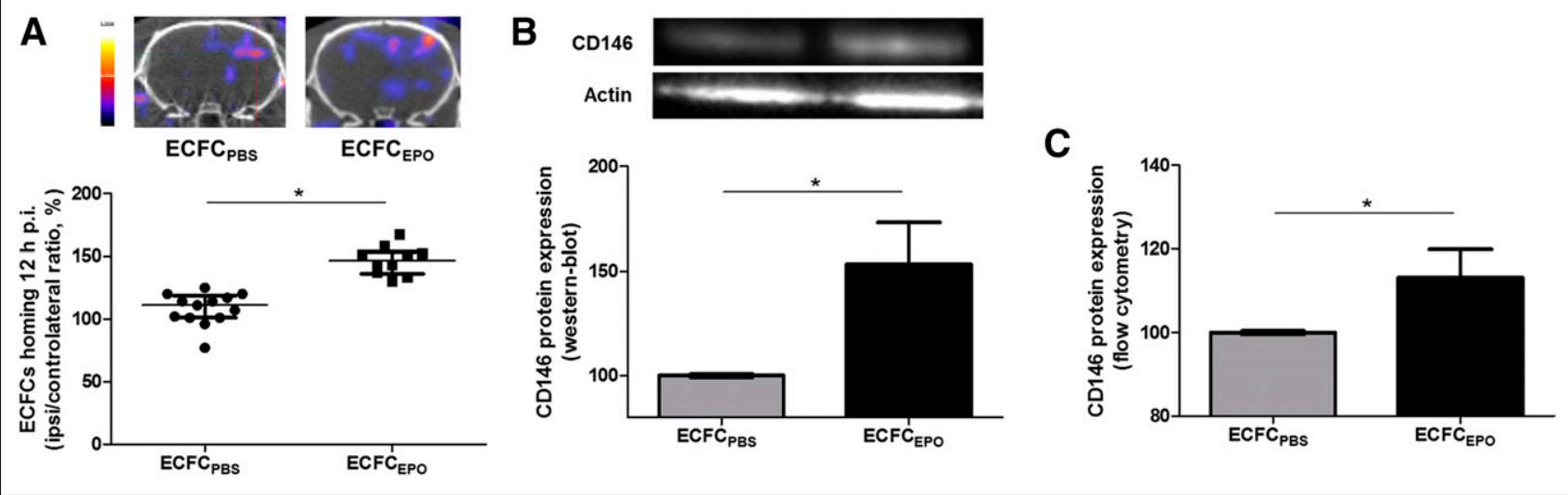

FIGURE 4. Effect of erythropoietin priming on ECFC homing and CD146 expression. (A) Representative brain tomographs and quantification (\% of

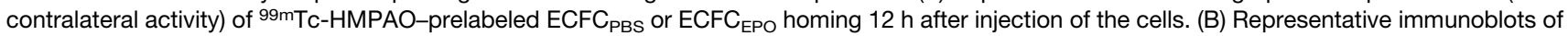
CD146 expression and quantification of ECFC lysates after $24 \mathrm{~h}$ of incubation with erythropoietin (\% of control). (C) Cytometry analysis quantifying CD146 expression on ECFCs after $24 \mathrm{~h}$ of incubation with erythropoietin (\% of control). ${ }^{*} P<0.05$. 


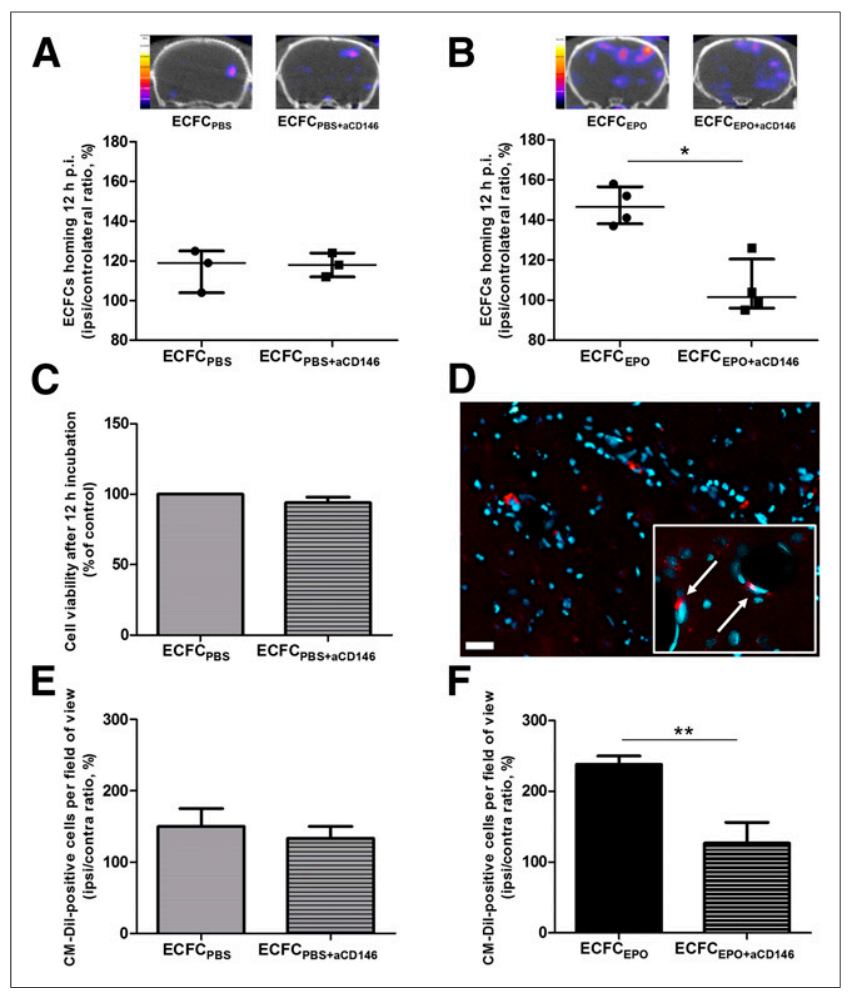

FIGURE 5. Effect of CD146 immunoblocking on ECFC homing. (A and B) Representative brain tomographs and quantification (\% of contralateral activity) of ${ }^{99 \mathrm{mTC}-\mathrm{HMPAO}-\text { prelabeled ECFC }}$ PBS $_{\text {or ECFC }} \mathrm{PBS}_{+\mathrm{aCD} 146}$ homing $(\mathrm{A})$ and $\mathrm{ECFC}_{\mathrm{EPO}}$ or ECFC $\mathrm{EPO}_{\mathrm{ECD} 146}$ homing $(\mathrm{B}) 12 \mathrm{~h}$ after injection of the cells. (C) ECFC viability after CD146 immunoblocking by flow cytometry (\% of control). (D) Representative brain immunofluores-

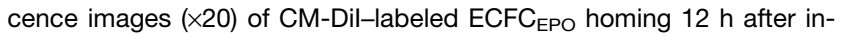
jection of the cells, with DAPI counterstaining in blue (scale bar, $20 \mu \mathrm{m}$ ). Inset shows $\times 40$ magnification of the cells (arrows) integrated to blood vessels. (E and F) Quantification (\% of contralateral activity) of CM-Dilpositive cells in ischemic hemisphere $12 \mathrm{~h}$ after ECFC PBS $_{\text {or }}$ $\mathrm{ECFC}_{\mathrm{PBS}+\mathrm{aCD} 146}$ injection (E) and after $\mathrm{ECFC}_{\mathrm{EPO}}$ or $\mathrm{ECFC}_{\mathrm{EPO}+\mathrm{aCD} 146}$ injection (F). ${ }^{\star} P<0.05$. ${ }^{\star \star} P<0.01$.

apoptosis was significantly and inversely correlated to ECFC homing $(r=-0.7251, P<0.0001)$ (Fig. 6B).

Fourteen days after MCAO, the ${ }^{99 \mathrm{~m}} \mathrm{Tc}-\mathrm{HMPAO} \mathrm{I} / \mathrm{C}$ ratio was higher in $\mathrm{ECFC}_{\mathrm{PBS}}(90 \% \pm 4 \% n=13, P<0.01)$ and $\mathrm{ECFC}_{\mathrm{EPO}}$ $(99 \% \pm 4 \%, n=10[P<0.001])$ than in control $(72 \% \pm 2 \% \%$, $n=10)$. The ${ }^{99 \mathrm{~m} T c-H M P A O ~} \mathrm{I} / \mathrm{C}$ ratio in $\mathrm{ECFC}_{\mathrm{EPO}}$ was significantly higher than that in $\mathrm{ECFC}_{\mathrm{PBS}}(P<0.05)$. CBF was significantly correlated to ECFC homing $(r=0.7348, P<0.0001)$ (Fig. 6C).

\section{DISCUSSION}

Various cell therapies after stroke, including the use of mesenchymal stem cells, cord blood cells, or endothelial progenitor cells, represent a potential innovative strategy with a wide therapeutic time window and are still under intensive preclinical and clinical evaluations $(11,12)$. We report here that erythropoietin priming of ECFCs enhanced the ability of cells to home to ischemic tissue and that the number of homed cells correlated with cerebral tissue recovery.

One limitation of our work consists in using immunocompetent rats with no immunosuppressive therapy, known to be neuroprotective (13-15). According to the BBB disruption, we could not exclude the involvement of peripheral cell immunity even if we previously excluded the lymphocytic inflammatory reaction (4) that could be explained by the low immunogenicity of cord blood cells $(16,17)$. Additionally, we previously reported that ECFCs did not proliferate and that only a few cells were visible $14 \mathrm{~d}$ after transplantation (4).

Early administration of exogenous ECFCs could anticipate the protective effect of endogenous mobilized endothelial progenitor cells after stroke (18) because mobilization occurs late, from 7 to $28 \mathrm{~d}$ after stroke, in humans (19). Moreover, several strategies aiming to circumvent the poor homing efficiency of cell-based therapies for stroke have already been explored, including arterial infusion (20), C-X-C chemokine receptor type 4 or vascular endothelial growth factor overexpression, or pharmacologic cotreatment $(6,21)$.

Erythropoietin, mainly known as the regulator of erythropoiesis, has been found to also induce nonhematopoietic effects (22) and contributes to the physiologic and pathologic angiogenesis of the body's innate response to tissue injury and especially to ischemic injury (23).

Here we reported that erythropoietin priming of ECFCs increased their homing ability and their benefit in animals up to $14 \mathrm{~d}$ after MCAO. Although trends were clearly visible, additional benefits of erythropoietin-primed ECFCs on neurofunctional recovery could not be demonstrated, probably because of the lack of sensitivity of clinical evaluation (24). However, brain SPECT/ CT gave us evidence of the therapeutic superiority of erythropoietin-primed ECFCs as shown by improved benefits in terms of BBB disruption, apoptosis activation, and CBF compared with unprimed ECFCs.

SPECT/CT is one of the most recommended imaging modalities for cell tracking $(25,26)$. We observed that erythropoietin priming of ECFCs increased their ability to home to ischemic tissues and potentiated their beneficial effect on BBB disruption, brain apoptosis, and CBF, as all of these correlated significantly with ECFC homing intensity. Our observations, in line with previous experiments on rodent models of hindlimb ischemia (5), underlie the major interest in ECFC homing-enhancing strategies in ischemic tissues after stroke.

Additionally, we observed that erythropoietin priming induced increased expression of ECFC CD146, a transmembrane immunoglobulin involved in ECFC adhesion to activated endothelium, migration, proliferation, and paracrine secretion (27-29). We observed that erythropoietin-induced CD146 overexpression mediated the increase in ECFC homing, as CD146 immunoblocking fully abrogated erythropoietin homing potentiation.

BBB disruption, peaking on day 2 after MCAO as we previously reported (7), is an early process involved in stroke physiopathology, consistent with the vasogenic edema reported after ischemic stroke in humans. Here we showed in vivo the benefits of ECFC injection on BBB disruption as assessed by ${ }^{99 m}$ Tc-DTPA imaging $2 \mathrm{~d}$ after MCAO. These results are in line with benefits observed from mesenchymal stem cell transplantation on BBB stabilization in rodent models of neuroinflammation (30). Additionally, we observed that benefits on BBB disruption were notably emphasized when cell homing was potentiated using erythropoietin-primed ECFCs. A correlation between BBB disruption and apoptosis in patients who experienced acute stroke is now proven (31) and places BBB disruption as a potential innovative target, which is in this case attenuated by transplanted ECFCs. 


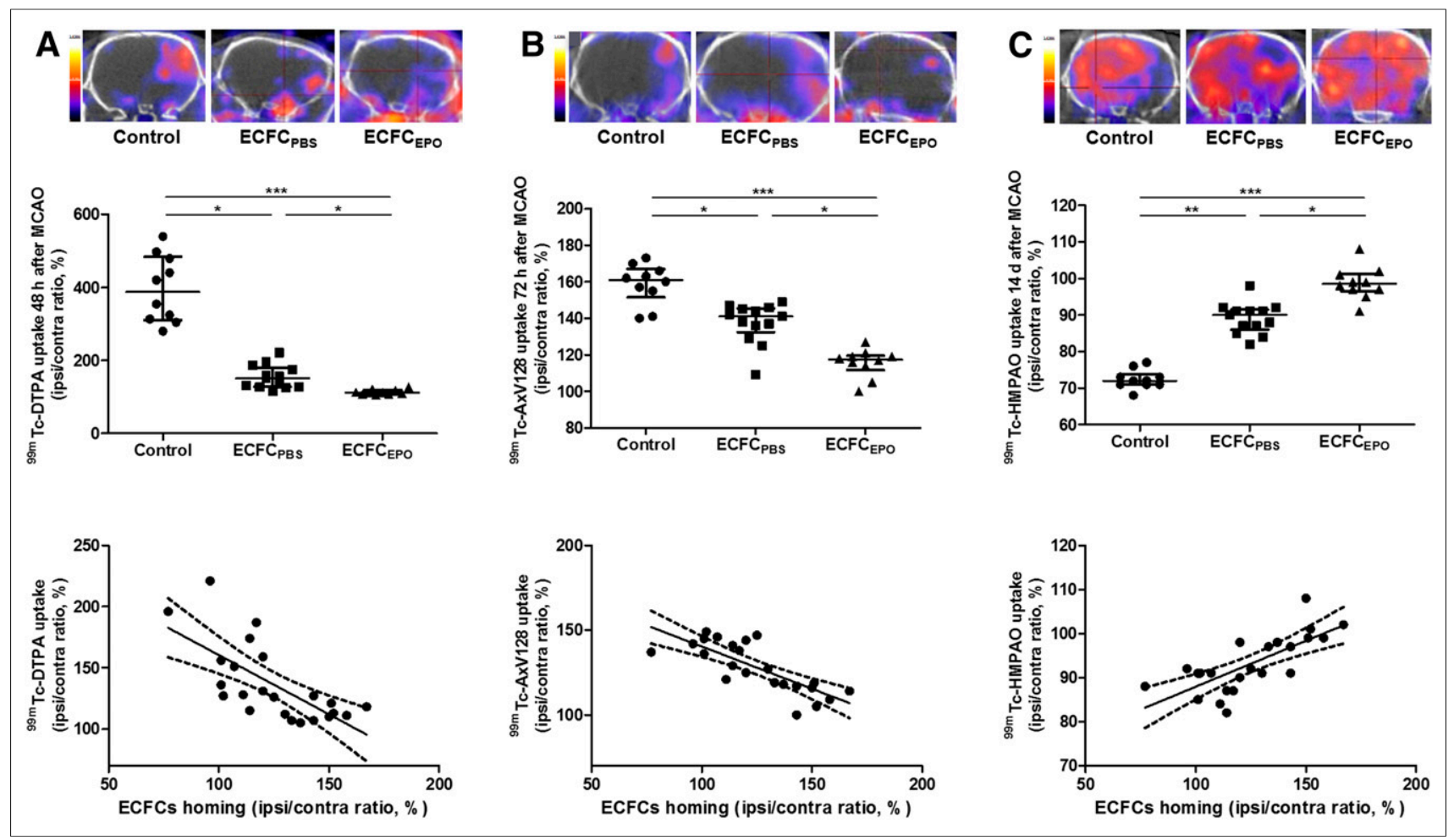

FIGURE 6. Effect of ECFC homing potentiation on BBB disruption (A), cerebral apoptosis (B), and CBF (C) on SPECT/CT performed using ${ }^{99 m T C-D T P A}$ $2 \mathrm{~d}$ after MCAO, 99mTc-annexin-V-128 $3 \mathrm{~d}$ after MCAO, and 99mTc-HMPAO $14 \mathrm{~d}$ after MCAO, respectively. (Top) Representative brain tomographs. (Middle) Quantification of activity in ischemic hemisphere (\% of contralateral activity). (Bottom) Correlation between ECFC homing and uptake (in A, B, and C: $r=-0.6930, P=0.0002 ; r=-0.7251, P<0.0001$; and $r=0.7348, P<0.0001$, respectively). ${ }^{\star} P<0.05$. ${ }^{\star \star} P<0.01$. ${ }^{\star \star \star} P<0.001$.

Clinical follow-up of apoptotic regions has already been correlated with neurologic outcome in patients (32). On day 3 after MCAO, we observed in $\mathrm{ECFC}_{\mathrm{PBS}}$ and $\mathrm{ECFC}_{\mathrm{EPO}}$ a huge decrease in apoptosis activation, matching previous ex vivo reports using bone-marrow-derived endothelial progenitor cells (33), $\mathrm{C}-\mathrm{X}-\mathrm{C}$ chemokine receptor type 4-primed endothelial progenitor cells (34), and vascular endothelial growth factor $\mathrm{R} 2^{+}$mesenchymal stem cell transplantation in MCAO rodent models (35). Moreover, we showed a direct correlation between the number of homing transplanted cells and the attenuation of apoptosis.

CBF assessed on day 14 after MCAO by $99 \mathrm{~m}$ Tc-HMPAO SPECT/ $\mathrm{CT}$, one of the modalities most described for experimental stroke imaging (36), allows definitive evaluation of tissue injury. We reported here a partial $\mathrm{CBF}$ restoration in the $\mathrm{ECFC}_{\mathrm{PBS}}$ animals that became nearly total in the $\mathrm{ECFC}_{\mathrm{EPO}}$ animals. This was probably obtained through an enhancement of ECFC angiogenic properties (5) and, according to the correlation between CBF and ECFC homing, partly through the potentiation of the cells' homing ability. The ECFC-mediated vascular regeneration mechanisms may imply metalloproteases (37), the stromal cell-derived factor $1 / \mathrm{C}-\mathrm{X}-\mathrm{C}$ chemokine receptor type 4 pathway (5), vascular endothelial growth factor secretion $(38)$, and interleukin 8 or $1 \beta(39,40)$ but also go through the increased CD146 expression on ECFCs (29).

\section{CONCLUSION}

Among the various strategies to enhance progenitor cell homing, we have shown that erythropoietin can be considered a powerful priming agent for ECFC-based therapy. Erythropoietin enhanced the benefits of ECFCs on BBB disruption, apoptosis, and CBF after MCAO by optimizing their ability to migrate to and engraft in the ischemic tissue, as was at least partially supported by CD146 expression. Finally, the correlations that were found between cell homing and BBB disruption, apoptosis, and CBF validate our hypothesis that increasing the homing ability of the transplanted cells may potentiate ischemic tissue recovery.

\section{DISCLOSURE}

The costs of publication of this article were defrayed in part by the payment of page charges. Therefore, and solely to indicate this fact, this article is hereby marked "advertisement" in accordance with 18 USC section 1734 . No potential conflict of interest relevant to this article was reported.

\section{ACKNOWLEDGMENTS}

We thank Sandrine Pons, Samantha Fernandez, Patricia Stellmann, Michel Skandalovski, and Samy Vigier for technical assistance; the Obstetrics and Gynecology Department of Conception Hospital (Prof. Claude D'Ercole and Prof. Chantal Gilbertas); and the cord-blood bank of Marseille (Prof. Christian Chabannon). Very special thoughts are extended to Prof. Pascale Pisano.

\section{REFERENCES}

1. Go AS, Mozaffarian D, Roger VL, et al. Heart disease and stroke statistics: 2014 update - a report from the American Heart Association. Circulation. 2014;129: e28-e292.

2. Li Y-F, Ren L-N, Guo G, et al. Endothelial progenitor cells in ischemic stroke: an exploration from hypothesis to therapy. J Hematol Oncol. 2015;8:33. 
3. Medina RJ, O’Neill CL, Sweeney M, et al. Molecular analysis of endothelial progenitor cell (EPC) subtypes reveals two distinct cell populations with different identities. BMC Med Genomics. 2010;3:18.

4. Moubarik C, Guillet B, Youssef B, et al. Transplanted late outgrowth endothelial progenitor cells as cell therapy product for stroke. Stem Cell Rev. 2011;7:208-220.

5. Bennis Y, Sarlon-Bartoli G, Guillet B, et al. Priming of late endothelial progenitor cells with erythropoietin before transplantation requires the CD131 receptor subunit and enhances their angiogenic potential. J Thromb Haemost. 2012;10:1914-1928.

6. Pellegrini L, Bennis Y, Guillet B, et al. Therapeutic benefit of a combined strategy using erythropoietin and endothelial progenitor cells after transient focal cerebral ischemia in rats. Neurol Res. 2013;35:937-947.

7. Garrigue P, Giacomino L, Bucci C, et al. Single photon emission computed tomography imaging of cerebral blood flow, blood-brain barrier disruption, and apoptosis time course after focal cerebral ischemia in rats. Int J Stroke. 2016;11:117-126.

8. Delorme B, Basire A, Gentile C, et al. Presence of endothelial progenitor cells, distinct from mature endothelial cells, within human CD146+ blood cells. Thromb Haemost. 2005;94:1270-1279.

9. Detante O, Moisan A, Dimastromatteo J, et al. Intravenous administration of ${ }^{99 \mathrm{~m}} \mathrm{Tc}-\mathrm{HMPAO}-$ labeled human mesenchymal stem cells after stroke: in vivo imaging and biodistribution. Cell Transplant. 2009;18:1369-1379.

10. Chen J, Sanberg PR, Li Y, et al. Intravenous administration of human umbilical cord blood reduces behavioral deficits after stroke in rats. Stroke. 2001;32:2682-2688.

11. Lee JS, Hong JM, Moon GJ, et al. A long-term follow-up study of intravenous autologous mesenchymal stem cell transplantation in patients with ischemic stroke. Stem Cells. 2010;28:1099-1106.

12. Hess DC, Sila CA, Furlan AJ, Wechsler LR, Switzer JA, Mays RW. A doubleblind placebo-controlled clinical evaluation of MultiStem for the treatment of ischemic stroke. Int J Stroke. 2014;9:381-386.

13. Labrande C, Velly L, Canolle B, et al. Neuroprotective effects of tacrolimus (FK506) in a model of ischemic cortical cell cultures: role of glutamate uptake and FK506 binding protein $12 \mathrm{kDa}$. Neuroscience. 2006;137:231-239.

14. Erlandsson A, Lin C-HA, Yu F, Morshead CM. Immunosuppression promotes endogenous neural stem and progenitor cell migration and tissue regeneration after ischemic injury. Exp Neurol. 2011;230:48-57.

15. Yuen C-M, Sun C-K, Lin Y-C, et al. Combination of cyclosporine and erythropoietin improves brain infarct size and neurological function in rats after ischemic stroke. J Transl Med. 2011;9:141.

16. Vendrame M, Gemma C, Pennypacker KR, et al. Cord blood rescues stroke-induced changes in splenocyte phenotype and function. Exp Neurol. 2006;199:191-200.

17. Roura S, Bagó JR, Soler-Botija C, et al. Human umbilical cord blood-derived mesenchymal stem cells promote vascular growth in vivo. PLoS One. 2012;7:e49447.

18. Mao L, Huang M, Chen S-C, et al. Endogenous endothelial progenitor cells participate in neovascularization via CXCR4/SDF-1 axis and improve outcome after stroke. CNS Neurosci Ther. 2014;20:460-468.

19. Zhou WJ, Zhu D, Yang G, et al. Circulating endothelial progenitor cells in Chinese patients with acute stroke. Hypertens Res. 2009;32:306-310.

20. Chen Y-L, Tsai T-H, Wallace CG, et al. Intra-carotid arterial administration of autologous peripheral blood-derived endothelial progenitor cells improves acute ischemic stroke neurological outcomes in rats. Int J Cardiol. 2015;201:668-683.

21. Bo C-J, Chen B, Jia R-P, et al. Effects of ischemic preconditioning in the late phase on homing of endothelial progenitor cells in renal ischemia/reperfusion injury. Transplant Proc. 2013;45:511-516.

22. Maiese K, Chong ZZ, Shang YC. Raves and risks for erythropoietin. Cytokine Growth Factor Rev. 2008;19:145-155.
23. Velly L, Pellegrini L, Guillet B, Bruder N, Pisano P. Erythropoietin 2nd cerebral protection after acute injuries: a double-edged sword? Pharmacol Ther. 2010;128:445-459.

24. Rosell A, Agin V, Rahman M, et al. Distal occlusion of the middle cerebral artery in mice: are we ready to assess long-term functional outcome? Transl Stroke Res. 2013;4:297-307.

25. Aicher A, Brenner W, Zuhayra M, et al. Assessment of the tissue distribution of transplanted human endothelial progenitor cells by radioactive labeling. Circulation. 2003;107:2134-2139.

26. Gavins FNE, Smith HK. Cell tracking technologies for acute ischemic brain injury. J Cereb Blood Flow Metab. 2015;35:1090-1099.

27. Bardin N, Anfosso F, Massé JM, et al. Identification of CD146 as a component of the endothelial junction involved in the control of cell-cell cohesion. Blood. 2001;98:3677-3684.

28. Bardin N, Blot-Chabaud M, Despoix N, et al. CD146 and its soluble form regulate monocyte transendothelial migration. Arterioscler Thromb Vasc Biol. 2009;29:746-753.

29. Kebir A, Harhouri K, Guillet B, et al. CD146 short isoform increases the proangiogenic potential of endothelial progenitor cells in vitro and in vivo. Circ Res. 2010;107:66-75.

30. Park HJ, Shin JY, Kim HN, Oh SH, Song SK, Lee PH. Mesenchymal stem cells stabilize the blood-brain barrier through regulation of astrocytes. Stem Cell Res Ther. 2015;6:187.

31. Lorberboym M, Blankenberg FG, Sadeh M, Lampl Y. In vivo imaging of apoptosis in patients with acute stroke: correlation with blood-brain barrier permeability. Brain Res. 2006;1103:13-19.

32. Zgavc T, Ceulemans A-G, Hachimi-Idrissi S, Kooijman R, Sarre S, Michotte Y. The neuroprotective effect of post ischemic brief mild hypothermic treatment correlates with apoptosis, but not with gliosis in endothelin-1 treated rats. BMC Neurosci. 2012;13:105.

33. Qiu J, Li W, Feng S, Wang M, He Z. Transplantation of bone marrow-derived endothelial progenitor cells attenuates cerebral ischemia and reperfusion injury by inhibiting neuronal apoptosis, oxidative stress and nuclear factor- $\mathrm{\kappa}$ expression. Int J Mol Med. 2013;31:91-98.

34. Chen J, Chen J, Chen S, et al. Transfusion of CXCR4-primed endothelial progenitor cells reduces cerebral ischemic damage and promotes repair in $\mathrm{db} / \mathrm{db}$ diabetic mice. PLoS One. 2012;7:e50105.

35. Bao X, Feng M, Wei J, et al. Transplantation of Flk-1+ human bone marrowderived mesenchymal stem cells promotes angiogenesis and neurogenesis after cerebral ischemia in rats. Eur J Neurosci. 2011;34:87-98.

36. Heiss W-D. Radionuclide imaging in ischemic stroke. J Nucl Med. 2014;55:1831-1841.

37. Morancho A, Ma F, Barceló V, Giralt D, Montaner J, Rosell A. Impaired vascular remodeling after endothelial progenitor cell transplantation in MMP9-deficient mice suffering cortical cerebral ischemia. J Cereb Blood Flow Metab. 2015;35:1547-1551.

38. Urbich C, Aicher A, Heeschen C, et al. Soluble factors released by endothelial progenitor cells promote migration of endothelial cells and cardiac resident progenitor cells. J Mol Cell Cardiol. 2005;39:733-742.

39. He T, Peterson TE, Katusic ZS. Paracrine mitogenic effect of human endothelial progenitor cells: role of interleukin-8. Am J Physiol Heart Circ Physiol. 2005;289:H968-H972.

40. Rosell A, Arai K, Lok J, et al. Interleukin- $1 \beta$ augments angiogenic responses of murine endothelial progenitor cells in vitro. J Cereb Blood Flow Metab. 2009;29:933-943. 